\title{
Standardization of grading embryo morphology
}

\author{
Catherine Racowsky • Michael Vernon • Jacob Mayer • G. David Ball • Barry Behr • \\ Kimball O. Pomeroy • David Wininger • William Gibbons • Joseph Conaghan • \\ Judy E. Stern
}

Received: 8 May 2010 / Accepted: 19 May 2010/Published online: 9 June 2010

(C) Springer Science+Business Media, LLC 2010

\begin{abstract}
Standardization of morphological assessment for embryo grading system was developed and is being implemented by the Society for Assisted Reproductive Technology (SART). A recent European consensus conference of embryologists from Europe and America is working toward adopting an embryo classification system modeled similarly to that of SART which, if adopted, would produce a de facto international standard to aid cross border collaboration.
\end{abstract}

Keywords Embryo · Grading · Evaluation .

Standardization $\cdot$ SART

Capsule An embryo grading system was developed and implemented by SART in 2006. A similar system is under development in Europe. International standardization of morphology assessments will aid cross border collaboration.

\section{Racowsky}

Department of Obstetrics and Gynecology,

Brigham \& Women's Hospital, Harvard Medical School,

Boston, MA, USA

\section{Vernon}

Department of Obstetrics and Gynecology,

West Virginia University,

Morgantown, VA, USA

J. Mayer

Jones Institute,

Norfolk, VA, USA

G. D. Ball

Seattle Reproductive Medicine,

Seattle, WA, USA

B. Behr

Department of Obstetrics and Gynecology, Stanford University,

Palo Alto, CA, USA

K. O. Pomeroy

Arizona Reproductive Medicine Specialists,

Phoenix, AZ, USA
We write with regards to implementation of international standards for grading and reporting embryo quality in assisted reproductive technology (ART) laboratories. This year marks a turning point in the history of grading preimplantation embryos. First, in the United States, the Society for Assisted Reproductive Technology (SART) mandated reporting of embryo grade. This resulted from efforts of a SART Embryo Morphology Subcommittee, comprised of embryologists and a clinician, who devised a simple standardized grading system that can be easily implemented in any ART laboratory. Second, last February,

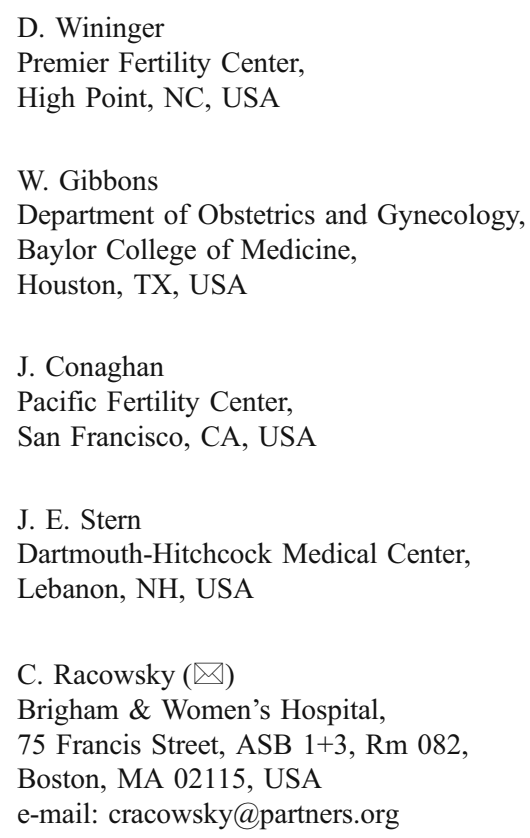


an initiative by the international society, Alpha, brought together a group of embryologists to garner consensus on embryo grading in Europe.

There have been numerous systems devised to grade and rank embryos (reviewed by [1, 2]). Simple grading systems assign one grade to account for the overall appearance of the cleavage stage embryo, taking into account cell symmetry and fragmentation [3]. More complex systems use a formula to predict pregnancy likelihood based on the appearance and development of an embryo [4]. More recent studies with cleavage stage embryos have evaluated the utility of assessing embryos on multiple days or only just before embryo transfer [5-7]. For embryos at the blastocyst stage, grading usually takes into account the morphology of the inner cell mass (ICM) and trophectoderm (TE), as well as the degree of expansion of the blastocyst cavity [8]. Despite the number and complexity of these and other grading systems, no convention exists for assigning alpha or numeric scores or for what represents a high or a low grade (reviewed by [9]).

Embryo morphology fields were added to the SART database in response to a request by SART member programs in 2004. This request was made due to concerns among the membership that despite SART discouraging inter-program success rate comparisons, such comparisons are made, and there was no direct way to benchmark differences in patient populations. Since embryo quality, as determined by morphology, bears a significant relation to implantation potential, it was decided that reporting of morphology fields could provide a mechanism to aid the assessment of patient reproductive potential. Moreover, it was felt that such collection would standardize grading systems among clinics, thereby improving quality assurance and quality improvement activities. Ultimately, this information may be used to reduce the number of embryos transferred and in turn lower the multiple pregnancy rates- including twin pregnancies. Recognizing the potential worth of standardizing embryo grading across SART member programs, the 2005 SART Executive Council charged our subcommittee with developing a grading system for the SART database (SARTCORS). The subcommittee realized that the embryo grading system must be 1) simple, 2) comprised of fields that have a basis in scientific inquiry with some proven predictive value, and 3) easily adopted in laboratories not routinely capturing these parameters. A three point grading system (Good, Fair and Poor) was developed and accompanied by a method for staging embryos using morphological features (Table 1).

Voluntary collection of the stage and grade of transferred embryos was initiated in June, 2006 for SART member clinics. Two analyses of the data, one involving 7,000 embryos, the other 70,000 , both provided robust associations between stage and grade of day 3 embryos with implantation and live birth rates [10,11], and collections into SARTCORS became mandatory as of March 2010. Although several other countries (such as the UK and Spain) already use standardized embryo grading systems, the Alpha consensus conference [12] recognized the need for standardization across Europe. We are pleased that these countries appear favorably disposed to utilization of a standardized system virtually identical to the SARTCORS system. Such international standardization should allow collaborative efforts across borders involving very large numbers of cycles, for the investigation of populationspecific questions regarding relationships among embryo morphology and cycle outcomes in the short-term, and health of the offspring in the long-term.

Conflict of Interest The authors can identify no potential conflicts of interest, neither financial nor any other, involved in the writing or publication of this manuscript.

Table 1 SART grading system

\begin{tabular}{lll}
\hline Growth phase & Overall grade & Stage \\
\hline Cleavage & Good, Fair, Poor & Cell \#: 1 through $>8$ \\
& & Fragmentation: $0 \%,<10 \%, 11-25 \%,>25 \%$ \\
& Symmetry: Perfect, Moderately Asymmetric, Severely Asymmetric \\
Morula & Good, Fair, Poor & Compaction: Complete, Incomplete \\
& & Fragmentation: $0 \%,<10 \%, 11-25 \%,>25 \%$ \\
Blastocyst & Good, Fair, Poor & Expansion: Early, Expanding, Expanded, Hatched \\
& & Inner Cell Mass: Good, Fair, Poor \\
& & Trophectoderm: Good, Fair, Poor \\
\end{tabular}




\section{References}

1. Boiso I, Veiga A, Edwards RG. Fundamentals of human embryonic growth in vitro and the selection of high-quality embryos for transfer. Reprod Biomed Online. 2002;5(3):328-50.

2. Gardner DK, Stevens J, Sheehan CB, Schoolcraft W. Analysis of blastocyst morphology. In: Elder K, Cohen J, editors. Human preimplantation embryo selection. London: Informa Healthcare; 2007. p. 79-87.

3. Veeck L. An atlas of human gametes and conceptuses. Carnforth: Parthenon Publishing; 1999.

4. Cummins J, Breen T, Harrison K, Shaw JM, Wilson LM, Hennessey JF. A formula for scoring human embryo growth rates in in vitro fertilization: its value in predicting pregnancy in comparison with visual estimates of embryo quality. J In Vitro Fert Embryo Transf. 1986;3(5):284-95.

5. Fisch J, Sher G, Adamowicz M, Keskintepe L. The graduated embryo score predicts the outcome of assisted reproductive technologies better than a single day 3 evaluation and achieves results associated with blastocyst transfer from day 3 embryo transfer. Fertil Steril. 2003;80:1352-8.

6. Chen C, Kattera S. Comparison of pronuclear zygote morphology and early cleavage stage status of zygotes as additional criteria in the selection of day 3 embryos: a randomized study. Fertil Steril. 2006;85:347-52.

7. Racowsky C, Ohno-Machado L, Kim J, Biggers JD. Is there an advantage to scoring embryos on more than 1 day? Hum Reprod. 2009;24(9):2104-13.

8. Gardner DK, Schoolcraft WB. In-vitro culture of human blastocysts. In: Jansen R, Mortimer D, editors. Towards reproductive certainty: fertility and genetics beyond. Carnforth: Parthenon Publishing; 1999. p. 378-88.

9. Ceyhan ST, Jackson KV, Racowsky C. Selecting the most competent embryo. In: Carrell DT, Racowsky C, Schlegel PN, Van Voorhis BJ, editors. Biennial review of infertility, vol 1. New York: Humana Press; 2009. p. 143-69.

10. Racowsky C, Stern JE, Gibbons WE, Behr B, Pomeroy KO, Biggers JD. National collection of embryo morphology data into SARTCORS: associations among cell number, fragmentation and blastomere asymmetry on day 3 with live birth rate. Fertil Steril. 2009;92(3, Suppl):S82, O-278.

11. Vernon M, Stern JE, Ball GD, Wininger D, Mayer J, Racowsky C. Utility of the national embryo morphology data collection by SART: Correlation between morphologic grade and live birth rate. Fertil Steril 2009; 92(3, Suppl):S164, P-270.

12. Joint Alpha/ESHRE Consensus Workshop on Embryo Assessment. Istanbul, Turkey; Feb 26, 2010. 